\title{
On a Question of Brézis and Korevaar Concerning a Class of Square-Summable Sequences
}

\author{
Richard Fournier* St. Ruscheweyh ${ }^{\dagger}$ \\ Luis Salinas ${ }^{\ddagger}$
}

CRM-3215

April 2006

\footnotetext{
*Département de Mathématiques et statistique et Centre de recherches mathématiques, Université de Montréal, C.P. 6128, succ. Centre-ville, Montréal, QC H3C 3J7, Canada; fournier@dms.umontreal.ca

${ }^{\dagger}$ Institut für Mathematik, Universität Würzburg, Würzburg, Germany; ruscheweyh@mathematik.uni-wuerzburg.de

‡Departamento de Informática, Universidad Técnica Federico Santa Maria, Valparaíso, Chile; lsalinas@inf.utfsm.cl
} 



\begin{abstract}
We give an elementary proof of a statement due to Brézis and Nirenberg: $\sum_{k=-\infty}^{\infty} k\left|a_{k}\right|^{2}$ is an integer whenever $\left\{a_{k}\right\}_{k=-\infty}^{\infty}$ is a sequence of complex numbers such that $\sum_{k=-\infty}^{\infty} a_{k} \bar{a}_{n+k}=\left\{\begin{array}{ll}0 & \text { if } n \neq 0, \\ 1 & \text { if } n=0,\end{array}\right.$ for all integers $n$ and $\sum_{k=-\infty}^{\infty}|k|\left|a_{k}\right|^{2}<\infty$.
\end{abstract}

Keywords and phrases: Fourier coefficients of unimodular functions, $H p$ spaces, Sobolev spaces

Mathematical Subject Classification: Primary 42A16; Secondary 30B10, 30A78 



\section{Introduction}

We consider sequences $\left\{a_{n}\right\}_{-\infty}^{\infty}$ of complex numbers such that

$$
\sum_{k=-\infty}^{\infty} a_{k} \bar{a}_{n+k}=\left\{\begin{array}{ll}
0 & \text { if } n \neq 0, \\
1 & \text { if } n=0,
\end{array} \text { for all integers } n\right.
$$

and

$$
\sum_{k=-\infty}^{\infty}|k|\left|a_{k}\right|^{2}<\infty
$$

Under these assumptions it has been proved by Brézis and Nirenberg [2, 3] that the sum of the series $\sum_{k=-\infty}^{\infty} k\left|a_{k}\right|^{2}$ is an integer, a rather unexpected and sparkling result. The motivation of Brézis and Nirenberg while proving this was to extend the notion of degree (i.e., index or winding number) to various classes of maps; their proof was rather indirect and used aspects of duality. In a remarkable paper [6] Korevaar studied what happens to the BrézisNirenberg result when the absolute convergence of the series in (2) is replaced by various notions of convergence of $\sum_{k=-\infty}^{\infty} k\left|a_{k}\right|^{2}$. In the same paper, Korevaar asked for a more direct proof of the result and the same question has been recently raised by Brézis during a talk at a meeting (2004) held in honour of Prof. Andrzej Granas on the occasion of his 75 th birthday. It is of course not so clear what is meant by a more direct proof. Our goal in this paper, let us say in a humble way, is to provide a different proof of the result based on facts more readily evident to complex analysts. Our work is also related to remarks of L. Boutet de Monvel and O. Gabber to be found in an appendix to the paper [1]. We shall finally also obtain the following

Theorem 1.1. Let $\left\{a_{k}\right\}_{0}^{\infty}$ be a (one-sided) sequence of complex numbers for which (1) and (2) hold. Then $B(z):=$ $\sum_{n=0}^{\infty} a_{k} z^{k}$ is a finite Blaschke product and the number of zeros of $B$ in the unit disc $\{z|| z \mid<1\}$, including multiplicities, is equal to $\sum_{k=1}^{\infty} k\left|a_{k}\right|^{2}$.

\section{Another Proof of the Brézis-Nirenberg Result}

We shall proceed by a number of lemmas.

Lemma 2.1. Under the hypothesis (1), the $2 \pi$-periodic function $u(\theta):=\sum_{k=-\infty}^{\infty} a_{k} e^{i k \theta}$ is well-defined and unimodular for almost all $\theta \in[0,2 \pi)$.

Proof. By the Riesz-Fischer theorem, there exists a $[0,2 \pi]$-square integrable function $u$ whose Fourier coefficients are the numbers $\left\{a_{n}\right\}_{n=-\infty}^{\infty}$ and by the famous result of Carleson, this function is almost everywhere equal to its Fourier series. (This may also be established by easing an older and weaker result of Fejér [7, p. 65]). In short, we may assume that $u(\theta):=\sum_{k=-\infty}^{\infty} a_{k} e^{i k \theta}$ is the Fourier series of a square summable function. We now define, for $0<r<1$,

$$
u_{r}(\theta)=\sum_{k=-\infty}^{\infty} a_{k} r^{|k|} e^{i k \theta}, \quad 0 \leq \theta<2 \pi
$$

This last series is absolutely and uniformly convergent. We have for each integer $n$,

$$
\begin{aligned}
\left.\left|\frac{1}{2 \pi} \int_{0}^{2 \pi} e^{i n \theta}\right| u_{r}(\theta)\right|^{2} d \theta-\frac{1}{2 \pi} \int_{0}^{2 \pi} & e^{i n \theta}|u(\theta)|^{2} d \theta \mid \\
& \leq \frac{1}{2 \pi} \int_{0}^{2 \pi}\left|u_{r}(\theta)^{2}-u(\theta)^{2}\right| d \theta \leq \frac{1}{2 \pi} \int_{0}^{2 \pi}\left(\left|u_{r}(\theta)\right|+|u(\theta)|\right)\left|u_{r}(\theta)-u(\theta)\right| d \theta \\
& \leq\left(\left(\frac{1}{2 \pi} \int_{0}^{2 \pi}\left|u_{r}(\theta)\right|^{2} d \theta\right)^{1 / 2}+\left(\frac{1}{2 \pi} \int_{0}^{2 \pi}|u(\theta)|^{2} d \theta\right)^{1 / 2}\right)\left(\frac{1}{2 \pi} \int_{0}^{2 \pi}\left|u_{r}(\theta)-u(\theta)\right|^{2} d \theta\right)^{1 / 2} \\
& \leq 2\left(\sum_{k=-\infty}^{\infty}\left|a_{k}\right|^{2}\right)^{1 / 2}\left(\sum_{k=-\infty}^{\infty}\left|a_{k}\right|^{2}\left(1-r^{2|k|}\right)\right)^{1 / 2}
\end{aligned}
$$

Since by Abel's continuity theorem

$$
\lim _{r \rightarrow 1} \sum_{k=-\infty}^{\infty}\left|a_{k}\right|^{2}\left(1-r^{2|k|}\right)=0
$$


we obtain

$$
\begin{aligned}
\frac{1}{2 \pi} \int_{0}^{2 \pi} e^{i n \theta}|u(\theta)|^{2} d \theta & =\lim _{r \rightarrow 1} \frac{1}{2 \pi} \int_{0}^{2 \pi} e^{i n \theta}\left|u_{r}(\theta)\right|^{2} d \theta, \\
& =\lim _{r \rightarrow 1} \frac{1}{2 \pi} \int_{0}^{2 \pi} e^{i n \theta}\left(\sum_{k=-\infty}^{\infty} a_{k} r^{|k|} e^{i k \theta}\right)\left(\sum_{k=-\infty}^{\infty} \bar{a}_{k} r^{\mid k} e^{-i k \theta}\right) d \theta \\
& =\lim _{r \rightarrow 1} \sum_{k=-\infty}^{\infty} a_{k} \bar{a}_{k+n} r^{|k|+|k+n|} \\
& =\sum_{k=-\infty}^{\infty} a_{k} \bar{a}_{k+n}= \begin{cases}0 & \text { if } n \neq 0, \\
1 & \text { if } n=0,\end{cases}
\end{aligned}
$$

the passage from (3) to (4) being justified by the absolute convergence of the Fourier series $u_{r}(\theta)$ while $(5)$ follows again from Abel's continuity theorem. This completes the proof of Lemma 2.1; this result may not be entirely new since a (weaker) version of it was stated without proof in a 1962 paper by Newman and Shapiro [9]. Moreover, the condition (1) is in fact equivalent to the unimodularity of the associated function $u(\theta)$. We may now write

$$
u(\theta)=e^{i U(\theta)}, \text { with } U(\theta) \in[0,2 \pi) \text { for almost all } \theta \in[0,2 \pi) .
$$

Our next result is

Lemma 2.2. Let $U(\theta) \sim \sum_{k=-\infty}^{\infty} b_{k} e^{i k \theta} ;$ then $\sum_{k=-\infty}^{\infty}|k|\left|b_{k}\right|^{2}<\infty$.

Proof. It is readily seen by the formula of Devinatz [10, p. 329] that

$$
4 \pi^{2} \sum_{k=-\infty}^{\infty}|k|\left|a_{k}\right|^{2}=\int_{0}^{2 \pi} \int_{0}^{2 \pi}\left|\frac{u(\theta)-u(\varphi)}{e^{i \theta}-e^{i \varphi}}\right|^{2} d \theta d \varphi
$$

$$
=\int_{0}^{2 \pi} \int_{0}^{2 \pi} \frac{\sin ^{2}((U(\theta)-U(\varphi)) / 2)}{\left|e^{i \theta}-e^{i \varphi}\right|^{2}} d \theta d \varphi<\infty .
$$

Moreover, by the same formula, we need to prove that

$$
\int_{0}^{2 \pi} \int_{0}^{2 \pi}\left|\frac{U(\theta)-U(\varphi)}{e^{i \theta}-e^{i \varphi}}\right|^{2} d \theta d \varphi<\infty .
$$

Because the function $U$ is bounded except possibly on a set of measure zero, we may choose a positive number $c$ so small that

$$
\int_{0}^{2 \pi} \int_{0}^{2 \pi} \frac{\sin ^{2}(c(U(\theta)-U(\varphi)) / 2)}{\left|e^{i \theta}-e^{i \varphi}\right|^{2}} d \theta d \varphi<\infty
$$

and

$$
|U(\theta)-U(\varphi)|^{2} \leq \frac{\pi^{2}}{c^{2}} \sin ^{2}\left(c \frac{U(\theta)-U(\varphi)}{2}\right) .
$$

Our claim then follows by comparison of (6), (7), and (8). We remark finally that our proof yields that, for some universal constants $c_{1}, c_{2}>0$,

$$
\begin{aligned}
c_{1} \int_{0}^{2 \pi} \int_{0}^{2 \pi}\left|\frac{f(\theta)-f(\varphi)}{e^{i \theta}-e^{i \varphi}}\right|^{2} d \theta d \varphi & \leq \int_{0}^{2 \pi} \int_{0}^{2 \pi}\left|\frac{F(\theta)-F(\varphi)}{e^{i \theta}-e^{i \varphi}}\right|^{2} d \theta d \varphi \\
& \leq c_{2} \int_{0}^{2 \pi} \int_{0}^{2 \pi}\left|\frac{f(\theta)-f(\varphi)}{e^{i \theta}-e^{i \varphi}}\right|^{2} d \theta d \varphi
\end{aligned}
$$

for all admissible functions $f$ unimodular and $2 \pi$-periodic with $f=e^{i F}$.

We shall now prove that the result of Brézis and Nirenberg holds for sequences $\left\{a_{n}\right\}_{n=-\infty}^{\infty}$ corresponding to smooth enough functions $u(\theta):=\sum_{k=-\infty}^{\infty} a_{k} e^{i k \theta}$.

Lemma 2.3. Let $u(\theta):=\sum_{k=-\infty}^{\infty} a_{k} e^{i k \theta}$ be as above with a continuous derivative $u^{\prime}(\theta)$ over $[0,2 \pi]$. Then $\sum_{k=-\infty}^{\infty} k\left|a_{k}\right|^{2}$ is an integer. 
Proof. Let $\phi=1 / 2 \pi i \int_{-\pi}^{\pi} \overline{u(t)} u^{\prime}(t) d t$. By using the functions $u_{r}(\theta)$ as before we obtain

$$
\phi=\sum_{k=-\infty}^{\infty} k\left|a_{k}\right|^{2} .
$$

Let us also define $\lambda(\theta):=\int_{-\pi}^{\theta} \overline{u(t)} u^{\prime}(t) d t$ and $\varphi(\theta):=u(\theta) e^{-\lambda(\theta)}$. Then by Lemma 2.1

$$
\varphi^{\prime}(\theta)=\left(1-|u(\theta)|^{2}\right) u^{\prime}(\theta) e^{-\lambda(\theta)} \equiv 0, \quad \theta \in[0,2 \pi] .
$$

We have that $\varphi(\theta) \equiv \varphi(-\pi)=u(-\pi)=u(\pi)$ and $e^{\lambda(\pi)}=e^{2 \pi i \phi}=1$. The claim follows.

We may now prove the general Brézis-Nirenberg result. Our arguments become clearer when expressed within the frame of certain Sobolev spaces (although we shall not use any of the deeper results concerning these spaces). Let $H^{1 / 2}$ denote the set of all $2 \pi$-periodic Fourier series $\sum_{k=-\infty}^{\infty} c_{k} e^{i k \theta}$ which satisfy $\sum_{k=-\infty}^{\infty}|k|\left|c_{k}\right|^{2}<\infty$. It is known [8, pp. 246-247] that $H^{1 / 2}$ can be turned into a Banach space when endowed with the norm

$$
\left\|\sum_{k=-\infty}^{\infty} c_{k} e^{i k \theta}\right\|:=\left(\sum_{k=-\infty}^{\infty}(|k|+1)\left|c_{k}\right|^{2}\right)^{1 / 2} .
$$

Let us consider a sequence $\left\{a_{n}\right\}_{-\infty}^{\infty}$ as above with $u(\theta):=\sum_{n=-\infty}^{\infty} a_{k} e^{i k \theta}=e^{i U(\theta)}$ where $U(\theta) \in[0,2 \pi)$ for almost all $\theta$ and $U(\theta):=\sum_{n=-\infty}^{\infty} a_{k} e^{i k \theta}$. Let also $U_{N}(\theta):=\sum_{k=-N}^{N} b_{k} e^{i k \theta}$ and $u_{N}(\theta):=e^{i U_{N}(\theta)}$. For the pseudo-norm

$$
\left\|\sum_{k=-\infty}^{\infty} c_{k} e^{i k \theta}\right\|_{p}:=\left(\sum_{k=-\infty}^{\infty}|k|\left|c_{k}\right|^{2}\right)^{1 / 2},
$$

we have

$$
\lim _{N \rightarrow \infty}\left\|U-U_{N}\right\|_{p}=\lim _{N \rightarrow \infty} \sum_{|k|>N}|k|\left|b_{k}\right|^{2}=0
$$

and by (9),

$$
\lim _{N \rightarrow \infty}\left\|u_{N} \bar{u}\right\|_{p}=\lim _{N \rightarrow \infty}\left\|e^{i\left(U-U_{N}\right)}\right\|_{p}=0 .
$$

By bounded convergence we know that

$$
\lim _{N \rightarrow \infty} \frac{1}{2 \pi} \int_{0}^{2 \pi} u_{N}(\theta) \overline{u(\theta)} d \theta=\lim _{N \rightarrow \infty} \frac{1}{2 \pi} \int_{0}^{2 \pi} e^{i\left(U_{N}(\theta)-U(\theta)\right)} d \theta=1
$$

and therefore

$$
\lim _{N \rightarrow \infty}\left\|u_{N} \bar{u}-1\right\|=0 \quad \text { and } \quad \lim _{N \rightarrow \infty} u_{N}\left(e^{i \theta}\right)=u\left(e^{i \theta}\right), \quad \text { a.e. }[0,2 \pi]
$$

Moreover,

$$
\begin{aligned}
\left\|u_{N}-u\right\|^{2}= & \left\|u\left(u_{N} \bar{u}-1\right)\right\|^{2} \\
= & \frac{1}{4 \pi^{2}} \int_{0}^{2 \pi} \int_{0}^{2 \pi}\left|\frac{u(\theta)\left(u_{N}(\theta) \bar{u}(\theta)-1\right)-u(\varphi)\left(u_{N}(\varphi) \bar{u}(\varphi)-1\right)}{e^{i \theta}-e^{i \varphi}}\right|^{2} d \theta d \varphi \\
& \quad+\frac{1}{2 \pi} \int_{0}^{2 \pi}\left|u_{N}(\theta) \bar{u}(\theta)-1\right|^{2} d \theta \\
\leq & \frac{1}{2 \pi^{2}} \int_{0}^{2 \pi} \int_{0}^{2 \pi}\left|u_{N}(\theta) \bar{u}(\theta)-1\right|^{2}\left|\frac{u(\theta)-u(\varphi)}{e^{i \theta}-e^{i \varphi}}\right|^{2} d \theta d \varphi \\
& \quad+\frac{1}{2 \pi^{2}} \int_{0}^{2 \pi} \int_{0}^{2 \pi}\left|\frac{u_{N}(\theta) \bar{u}(\theta)-u_{N}(\varphi) \bar{u}(\varphi)}{e^{i \theta}-e^{i \varphi}}\right|^{2} d \theta d \varphi \\
& \quad+\frac{1}{2 \pi} \int_{0}^{2 \pi}\left|u_{N}(\theta) \bar{u}(\theta)-1\right|^{2} d \theta \\
= & \frac{1}{2 \pi^{2}} \int_{0}^{2 \pi} \int_{0}^{2 \pi}\left|u_{N}(\theta) \bar{u}(\theta)-1\right|^{2}\left\{\left|\frac{u(\theta)-u(\varphi)}{e^{i \theta}-e^{i \varphi}}\right|^{2}+\pi\right\} d \varphi d \theta+2\left\|u_{N} \bar{u}\right\|_{p} .
\end{aligned}
$$

By (10), (11) and bounded convergence, we obtain $\lim _{N \rightarrow \infty}\left\|u_{N}-u\right\|=0$, i.e. the unimodular function $u$ is the $H^{1 / 2}$-limit of smooth unimodular functions $u_{N}$. Let us write $u_{N}(\theta):=\sum_{k=-\infty}^{\infty} a_{k}(N) e^{i k \theta}$; we know that $\left\|u_{N}\right\|$ 
is bounded above (because $\lim _{N \rightarrow \infty}\left\|u_{N}\right\|=\|u\|$ ) and the sequence of integers $\sum_{k=-\infty}^{\infty} k\left|a_{k}(N)\right|^{2}, N=1,2, \ldots$ is therefore also bounded above; we may therefore assume that for $N$ large enough, $\sum_{k=-\infty}^{\infty} k\left|a_{k}(N)\right|^{2}:=k_{u}$ is a fixed integer depending only on $u$. Further

$$
\begin{aligned}
\sum_{k=-\infty}^{\infty} k\left|a_{k}(N)-a_{k}\right|^{2} & =-\sum_{k=-\infty}^{\infty} k\left|a_{k}(N)\right|^{2}+\sum_{k=-\infty}^{\infty} k\left|a_{k}\right|^{2}-2 \sum_{k=-\infty}^{\infty} k \bar{a}_{k}(N)\left(a_{k}-a_{k}(N)\right) \\
& =-k_{u}+\sum_{k=-\infty}^{\infty} k\left|a_{k}\right|^{2}-2 \sum_{k=-\infty}^{\infty} k \bar{a}_{k}(N)\left(a_{k}-a_{k}(N)\right)
\end{aligned}
$$

where

$$
\left|\sum_{k=-\infty}^{\infty} k\right| a_{k}(N)-\left.a_{k}\right|^{2} \mid \leq\left\|u_{N}-u\right\|
$$

and

$$
\begin{aligned}
\left|2 \sum_{k=-\infty}^{\infty} k \bar{a}_{k}(N)\left(a_{k}-a_{k}(N)\right)\right| \leq 2 \sum_{k=-\infty}^{\infty}|k|\left|a_{k}(N)\right|\left|a_{k}-a_{k}(N)\right| & \\
& \leq 2\left(\sum_{k=-\infty}^{\infty}|k|\left|a_{k}(N)\right|^{2}\right)^{1 / 2}\left(\sum_{k=-\infty}^{\infty}|k|\left|a_{k}-a_{k}(N)\right|^{2}\right)^{1 / 2} \\
& \leq 2\left\|u_{N}\right\|\left\|u-u_{N}\right\| \\
& \leq 2(\|u\|+1)\left\|u-u_{N}\right\| .
\end{aligned}
$$

We clearly have

$$
\sum_{k=-\infty}^{\infty} k\left|a_{k}\right|^{2}=k_{u}=\sum_{k=-\infty}^{\infty} k\left|a_{k}(N)\right|^{2}, \quad \text { for all } N \text { large enough. }
$$

\section{Proof of Theorem 1.1}

We write $W(z):=\sum_{k=0}^{\infty} a_{k} z^{k}$ for $z \in \mathbb{D}:=\{z|| z \mid<1\}$. Because $\sum_{k=0}^{\infty}\left|a_{k}\right|^{2}<\infty, W$ is well-defined and in fact belongs to the Hardy space $H_{2}(\mathbb{D})$ (see [5] for a standard reference concerning Hardy spaces). By Lemma 2.1, the radial limits (which are known to exist a.e. $[0,2 \pi)$ )

$$
W\left(e^{i \theta}\right):=\lim _{\substack{r \rightarrow 1 \\ 0<r<1}} W\left(r e^{i \theta}\right)
$$

satisfy $\left|W\left(e^{i \theta}\right)\right|=1$ almost everywhere, i.e., $W$ is an inner function and as such can be factored as

$$
W(z)=B(z) I(z)
$$

where $B$ is a Blaschke product and $I$ a singular inner function,

$$
I(z)=e^{-1 / 2 \pi \int_{0}^{2 \pi}\left(1+z e^{i \theta}\right) /\left(1-z e^{i \theta}\right) d \mu(\theta)}
$$

with $\mu$ increasing on $[0,2 \pi], \mu^{\prime}(\theta)=0$ for almost all $\theta$. If $W$ is not a finite product, it is known that $W$ shall take all values in the unit disc $\mathbb{D}$ infinitely many times, except possibly for an exceptional set of planar measure zero (this is due to Frostman [4, p. 35]). In other words,

$$
\begin{aligned}
\infty & =\lim _{r \rightarrow 1} \iint_{x^{2}+y^{2} \leq r^{2}}\left|W^{\prime}(x+i y)\right|^{2} d x d y \\
& =\lim _{r \rightarrow 1} \frac{1}{2} \int_{0}^{2 \pi} r e^{i \theta} W^{\prime}\left(r e^{i \theta}\right) \overline{W\left(r e^{i \theta}\right)} d \theta \\
& =\lim _{r \rightarrow 1} \pi \sum_{n=1}^{\infty} n\left|a_{n}\right|^{2} r^{2 n} .
\end{aligned}
$$

This of course contradicts the hypotheses (2), again because of the Abel continuity theorem. We may therefore assume that $W$ is a constant of modulus 1 or else a finite Blaschke product,

$$
W(z)=\prod_{j=1}^{J} \frac{z-a_{j}}{1-\bar{a}_{j} z}, \quad\left|a_{j}\right|<1, j=1,2, \ldots
$$


and then

$$
\begin{aligned}
\sum_{n=1}^{\infty} n\left|a_{n}\right|^{2} & =\frac{1}{2 \pi} \int_{0}^{2 \pi} e^{i \theta} W^{\prime}\left(e^{i \theta}\right) \overline{W\left(e^{i \theta}\right)} d \theta \\
& =\frac{1}{2 \pi i} \int_{|\zeta|=1} \frac{W^{\prime}(\zeta)}{W(\zeta)} d \zeta \\
& =J
\end{aligned}
$$

by the argument principle.

\section{References}

[1] A. Boutet de Monvel-Berthier, V. Georgescu, and R. Purice, A boundary value problem related to the GinzburgLandau model, Comm. Math. Phys. 142 (1991), no. 1, 1-23.

[2] H. Brézis and L. Nirenberg, Degree theory and BMO. I. Compact manifolds without boundaries, Selecta Math. (N.S.) 1 (1995), no. 2, 197-263.

[3] _ Degree theory and BMO. II. Compact manifolds with boundaries, Selecta Math. (N.S.) 2 (1996), no. 3, 309-368.

[4] E. F. Collingwood and A. J. Lohwater, The theory of cluster sets, Cambridge Univ. Press, Cambridge, 1966.

[5] P. Koosis, Introduction to Hp spaces, Cambridge Univ. Press, Cambridge, 1998.

[6] J. Korevaar, On a question of Brézis and Nirenberg concerning the degree of circle maps, Selecta Math. (N.S.) 5 (1999), no. 1, 107-122.

[7] E. Landau and D. Gaier, Darstellung und Begründung einiger neuerer Ergebnisse der Funktionentheorie (German) [Presentation and explanation of some more recent results in function theory], 3rd ed., Springer-Verlag, Berlin, 1986.

[8] R. C. McOwen, Partial differential equations, Prentice Hall, Upper Saddle River, 2003.

[9] D. J. Newman and H. S. Shapiro, The Taylor coefficients of inner functions, Michigan Math. J. 9 (1962), 249-255.

[10] B. Simon, Orthogonal polynomials on the unit circle. I. Classical theory, Amer. Math. Soc. Colloq. Publ., vol 54, Part 1, Amer. Math. Soc., Providence, R.I., 2005. 